\title{
Proposal of new syntaxonomic classification of Myriophyllo- -Nupharetum W. Koch 1926 phytocenoses and their distribution in Poland
}

H. TOMASZEWICZ

Institute of Botany, Warsaw University, Warsaw

(Received: February 3, 1977)

\begin{abstract}
The author gives a new syntaxonomic classification of the phytocenoses included in the association Myriophyllo-Nupharetum described by W. $\mathrm{Koch}$ (1926) without any documentation material. After analysing 1554 phytosociological records from the territory of Poland, in which species considered as characteristic for Myriophyllo-Nupharetum (Nuphar luteum, Nymphaea alba, N. candida and Myriophyllum verticillatum) were the forming species (in this case also the dominating ones), it was considered necessary to classify these phytocenoses to three separate associations: Nuphareto-Nymphaeetum albae, Nymphaeetum candidae and Myriophylletum verticillati. A table representing the association Nuphareto-Nymphaeetum albae, 3 synthetic tables and maps of distribution of the so far recorded sites or groups of sites of the above distinguished units in Poland are attached.
\end{abstract}

The association Myriophyllo-Nupharetum (Myriophylleto verticillati-Nupharetum W. K o c h 1926) mentioned in nearly all monographic and synthetic works concerning aquatic vegetation has been described by $\mathrm{K} \mathrm{o} \mathrm{c} \mathrm{h} \mathrm{(1926)} \mathrm{without} \mathrm{any} \mathrm{documentation} \mathrm{material} \mathrm{in} \mathrm{the} \mathrm{form} \mathrm{of} \mathrm{phyto-}$ sociological records or synthetic tables. Therefore this syntaxon to which $\mathrm{K}$ o c h only gave a name with a very summary characteristic was (and still is) variously interpreted by numerous authors. It is sufficient to peruse several monographs dealing with aquatic communities to realise what phytocenoses are included in this association. Beside phytocenoses built by Nuphar luteum, Nymphaea alba, N. candida and Myriophyllum verticillatum, namely, which are generally mentioned as species characteristic for Myriophyllo-Nupharetum, phytocenoses are included with such dominant species as: Myriophyllum spicatum, Elodea canadensis, Ceratophyllum demersum, Batrachium circinatum, Potamogeton natans, 
Trapa natans, Nuphar pumilum, Polygonum amphibium f. natans. In these phytocenoses frequently the species characteristic for Myriophyllo-Nupharetum are lacking or they are very scarce. Such a state of affairs can hardly continue longer with the present knowledge of water communities and their ecology. Within the same association, namely, phytocenoses are grouped with a completely differen species composition, different dynamics, ecological amplitudes and habitat requirements, which in the system of plant communities represent two separate alliances (Nymphaeion and Eu-Potamion).

Interesting data were supplied by analysis of the phytocenoses built by species considered as characteristic for Myriophyllo-Nupharetum. The phytocenoses which were included (and sometimes still are) into the community Myriophyllo-Nupharetum (arbitrarily by the authors) and which already have their own syntaxons and a place in the system of plant communities as for instance Elodeetum canadensis (Pign. 1953) Pass. 1964, Ceratophylletum demersi Hild 1956, Nupharetum pumili Oberd. 1957, Trapetum natantis Müller et Görs 1960, Potametum natantis Soó 1927, Ranunculetum circinati (Bennema et Westh. 1943) Segal 1956, Polygonetum natantis Soó 1927, Myriophylletum spicati Soó 1927.

Myriophyllum verticillatum is a typical immersed plant developing optimally beyond the range (depth) of occurrence of vegetation with floating leaves and it belongs, like other species of immersed plants, to the species characteristic for the alliance Eu-Potamion. Thus, it cannot be the species characteristic for Myriophyllo-Nupharetum, an association belonging to the alliance Nymphaeion. It is, therefore, correct that the associations Myriophylletum verticillati Soó 1927 was distinguished including phytocenoses with dominant Myriophyllum verticillatum. On the other hand, Nuphar luteum, Nymphaea alba and N. candida are typical plants with floating leaves, the ecological amplitudes of which overlap to a smaller or larger extent.

In order to establish the contribution of the particular species reported as characteristic for Myriophyllo-Nupharetum in the phytocenoses built by them and to find what floristic combinations they form, 1268 phytosociological records from published papers and unpublished material from the entire territory of Poland were analysed. It appeared that 11 different floristic combinations occur in the phytocenoses. From among the 1268 records Nuphar luteum was the dominant species only in 446, in 67 it was Nymphaea alba, in 337 - Nuphar luteum and Nymphaea alba, in $43-$ Myriophyllum verticillatum, in $144-$ Nuphar luteum and Myriophyllum verticillatum, in $17-$ Nymphaea alba and Myriophyllum verticillatum, in 18 - Nymphaea candida, in $72-$ Nymphaea candida and Nuphar luteum, in $3-N y m p h a e a$ alba, N. candida and Nuphar luteum, in 99 - Nuphar luteum, Nymphaea alba and Myriophyllum verticillatum, in 22 - Nuphar luteum, Nymphaea candi- 
$d a$ and Myriophyllum verticillatum. There are no phytocenoses where only Nymphaea alba and $N$. candida would be the dominant species, neither are there any composed of Nymphaea candida and Myriophyllum verticillatum. It should also be stressed that in about 90 per cent of the phytocenoses where Nuphar luteum and Myriophyllum verticillatum cccurred, the building (dominant) species was Nuphar luteum. The situation is similar as regards phytocenoses formed by Nymphaea alba and Myriophyllum verticillatum. Here too about 90 per cent of the patches is built of Nymphaea alba. In phytocenoses with 3 characteristic species the prevailing species are Nuphar luteum and Nymphaea alba also in about 90 per cent of these communities. It results from the foregoing data that about 88 per cent of all phytocenoses with dominant species characteristic at the same time for Myriophyllo-Nupharetum consist of Nuphar luteum and Nymphaea alba. Phytocenoses in which Myriophyllum verticillatum is the dominant species constitute about 7,5 per cent of the analysed phytocenoses. It should be mentioned that in the general list Myriophyllum verticillatum occurs only in 17 per cent of the records (class I of constancy) of phytocenoses built by Nuphar luteum and Nymphaea alba. This confirms the weak association of this species with these communities. Phytocenoses in which the dominant species is Nymphaea candida constitute about 5 per cent of the analysed phytosociological record material. These phytocenoses have a somewhat different character and cannot be included into the same association with those built by Nymphaea alba and Nuphar luteum because N. alba and $N$. candida exclude one another and do not build any* communities together in which both these species would occur in equal quantities. N e uhäusl (Seg a l, 1968) among others calls attention to this fact.

It results from the above mentioned data that phytocenoses built by Myriophyllum verticillatum should be included into the association Myriophylletum verticillati Soó 1927 within the alliance Eu-Potamion. On the other hand, phytocenoses formed by Nymphaea candida constitute, as compared with the phytocenoses built by Nuphar luteum and Nymphaea alba, a distinct type of vegetation and should be classified to the association Nymphaeetum candidae Miljan 1958 within the alliance Nymphaeion. In view of the separation of the associations Myriophylletum verticillati and Nymphaeetum candidae, there remain as characteristic species of the Myriophyllo-Nupharetum phytocenoses only Nuphar luteum and Nymphaea alba. Since neither Myriophyllum verticillatum nor M. spicatum can be characteristic species for Myriophyllo-Nupharetum, the syntaxonomic unit including phytocenoses formed by Nuphar luteum and Nymphaea alba has a different diagnosis and cannot be named Myriophyllo-Nupharetum. I therefore suggest to adopt an unequivocal characteristic for the Myriophyllo-Nupharetum "reduced" in this way and give it a new name - Nuphareto-Nymphaeetum albae. 


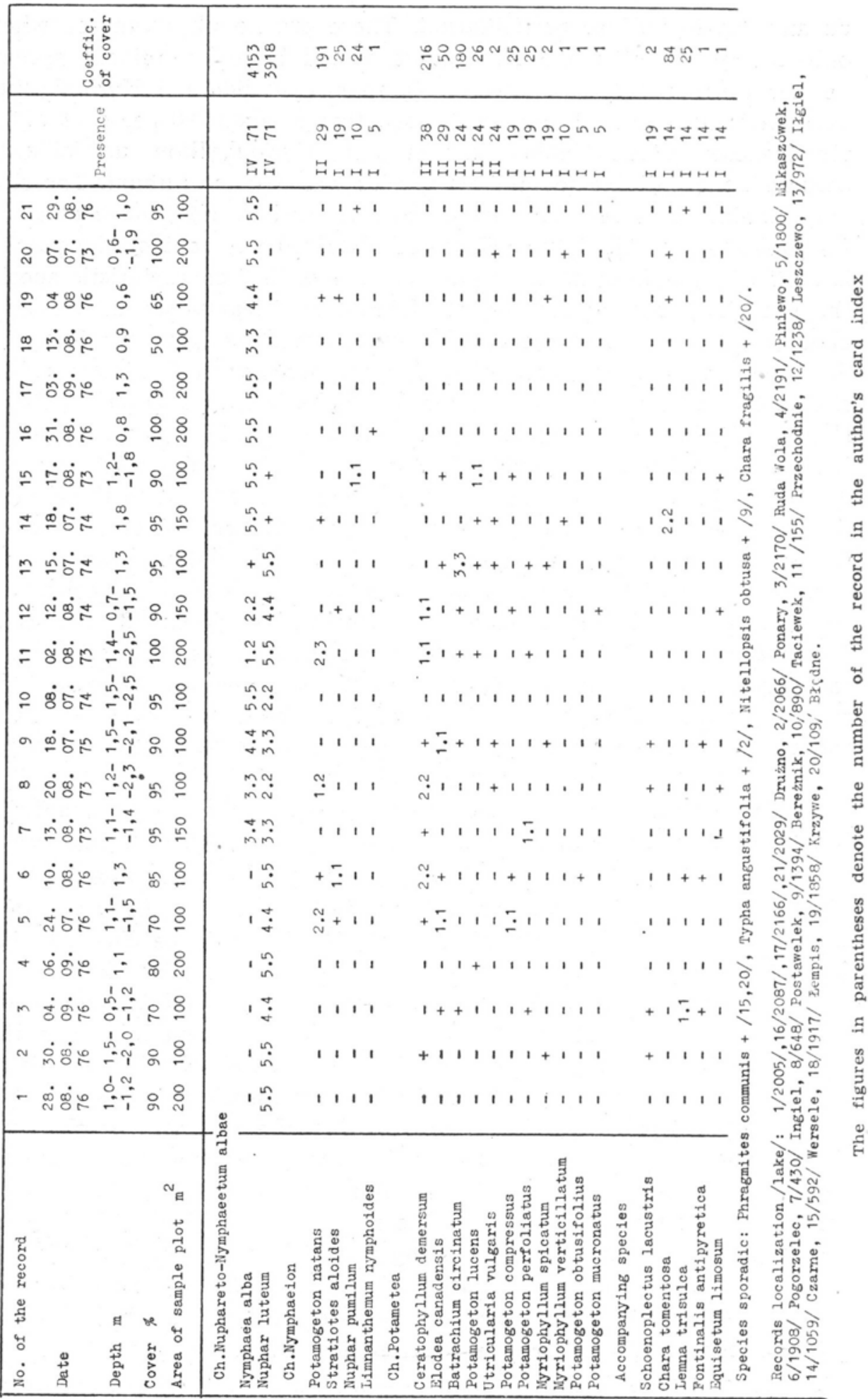


Table 2

Nuphareto-Nymphaeetum albae ass. nova

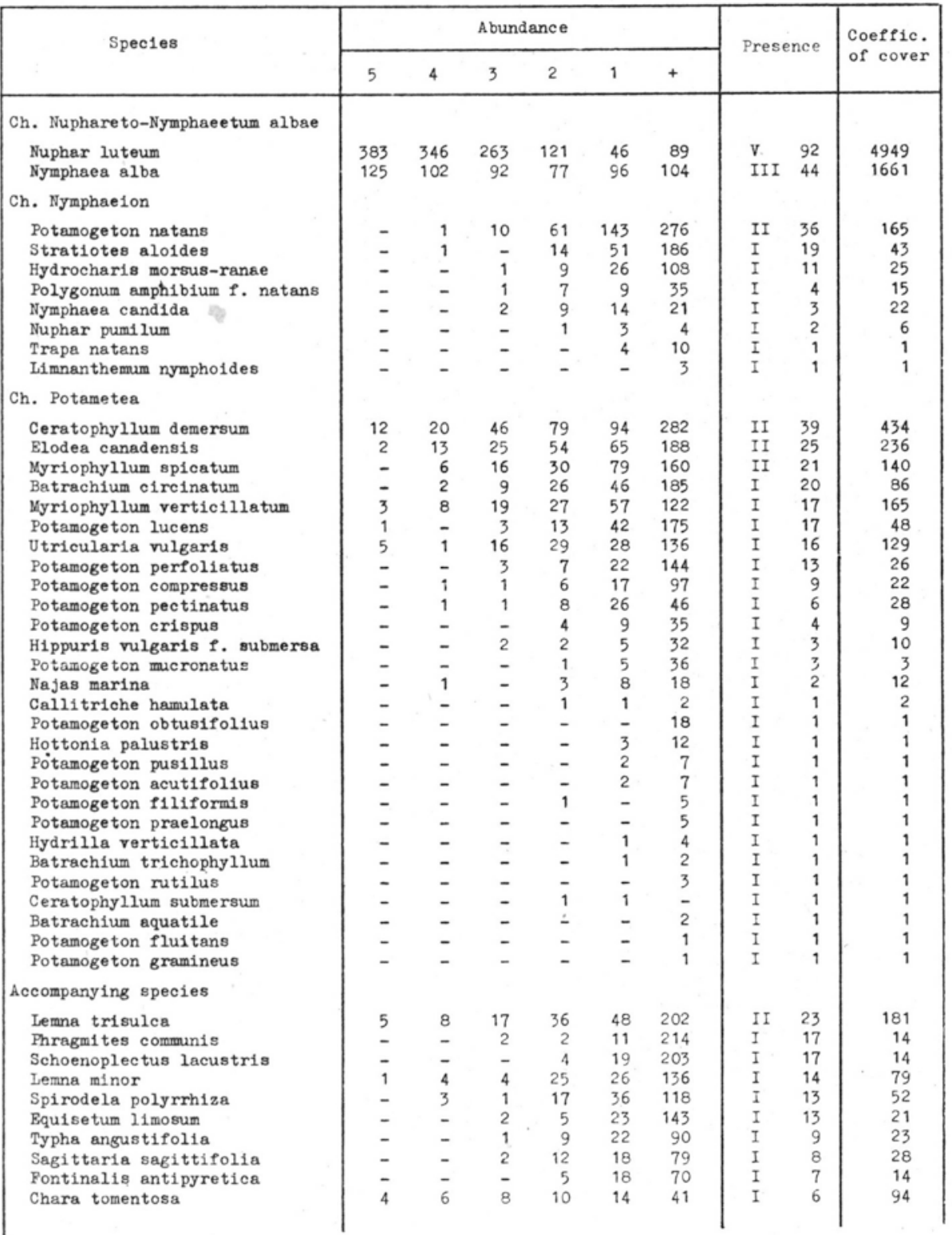

The figures in columns $5-+$ denote the absolute incidence

The characteristic dominant species constituting this syntaxon would be only Nuphar luteum and Nymphaea alba. The attached phytosociological table (Table 1) groups in various ways the developed Nuphareto-Nymphaeetum albae phytocenoses. Nuphar luteum forms phytocenoses on all types of habitats on which Nymphaea alba occurs, whereas, the latter 
Table 3

Myriophylletum vertic1llati Soó 1927

\begin{tabular}{|c|c|c|c|c|c|c|c|c|c|}
\hline \multirow{2}{*}{ Spec1es } & \multicolumn{6}{|c|}{ Abundance } & \multirow{2}{*}{\multicolumn{2}{|c|}{ Presence }} & \multirow{2}{*}{$\begin{array}{l}\text { Coeffic. } \\
\text { of cover }\end{array}$} \\
\hline & 5 & 4 & 3 & 2 & 1 & + & & & \\
\hline \multicolumn{10}{|l|}{ Ch. Myriophylletum verticillati } \\
\hline Myriophyllum verticillatum & 68 & 22 & 25 & - & - & - & $\mathrm{V}$ & 100 & 7185 \\
\hline \multicolumn{10}{|l|}{ Ch. Bu-Potamion } \\
\hline Batrachium circinatum & - & - & 4 & 4 & 8 & 29 & II & 39 & 229 \\
\hline Ceratophyllum demersum & - & - & 8 & 3 & 2 & 29 & II & 37 & 318 \\
\hline Elodea canadensis & - & 3 & 5 & 4 & 11 & 12 & II & 30 & 436 \\
\hline Potamogeton lucens & - & - & - & 1 & 7 & 14 & $\mathrm{I}$ & 19 & 47 \\
\hline Myriophyllum spicatum & - & - & - & - & 4 & 12 & I & 14 & 18 \\
\hline Potamogeton compressus & - & - & 2 & 1 & - & 5 & I & 7 & 81 \\
\hline Potamogeton perfollatus & - & - & - & 1 & 2 & 5 & 2 & 7 & 24 \\
\hline Potamogeton pusillus & - & 1 & 1 & 3 & 1 & 1 & I & 6 & 137 \\
\hline Potamogeton pectinatus & - & - & - & 2 & 1 & 2 & I & 4 & 35 \\
\hline Potamogeton mucronatus & - & - & - & 1 & - & 3 & I & 3 & 15 \\
\hline Potamogeton crispus & - & - & - & - & 1 & 2 & I & 3 & 5 \\
\hline Hippuris vulgaris $f$. submerga & - & - & - & - & - & 3 & $\mathrm{I}$ & 3 & 1 \\
\hline Potamogeton obtusifolius & - & - & - & - & - & 2 & $\mathrm{I}$ & 2 & 1 \\
\hline Ceratophyllum submersum & - & - & - & 1 & - & - & I & 1 & 15 \\
\hline Potamogeton acutifolius & - & - & - & - & - & 1 & I & 1 & 1 \\
\hline Hydrilla verticillata & - & - & - & - & - & 1 & I & 1 & 1 \\
\hline Potamogeton fillformis & - & - & - & - & - & 1 & $\mathrm{I}$ & 1 & 1 \\
\hline Potamogeton praelongus & - & - & - & - & - & 1 & I & 1 & 1 \\
\hline \multicolumn{10}{|l|}{ Ch. Potametea } \\
\hline Nuphar luteum & - & - & 9 & 11 & 16 & 19 & III & 48 & 532 \\
\hline Potamogeton natans & - & - & 4 & 3 & 5 & 38 & III & 43 & 201 \\
\hline Nymphaea alba & - & - & - & 1 & 11 & 19 & II & 27 & 65 \\
\hline Utricularia vulgaris & - & - & - & 3 & 4 & 16 & I & 20 & 64 \\
\hline Stratiotes aloides & - & - & - & 3 & 4 & 14 & $\mathrm{I}$ & 18 & 64 \\
\hline Hydrocharis morsus-ranae & - & - & 2 & 3 & 4 & 6 & I & 13 & 129 \\
\hline Hottonia palustris & - & - & - & 3 & 1 & - & I & 3 & 50 \\
\hline Nuphar pumilum & - & - & - & - & 3 & 1 & I & 3 & 13 \\
\hline Polygonum amphibium $f$. natans & - & - & - & - & 2 & 2 & $\mathrm{I}$ & 3 & 9 \\
\hline Potamogeton fluitans & - & - & 1 & 1 & - & 1 & I & 3 & 48 \\
\hline Batrachium aquatile & _- & - & - & 1 & - & 1 & I & 2 & 15 \\
\hline Nymphaea cand1da & - & - & - & - & - & 2 & $\mathrm{I}$ & 2 & 1 \\
\hline Callitriche polymorpha & - & - & - & 1 & - & - & $\mathrm{I}$ & 1 & 15 \\
\hline Batrachium trichophyllum & - & - & - & - & 1 & - & I & 1 & 4 \\
\hline \multicolumn{10}{|l|}{ Accompanying species } \\
\hline Lemna trisulca & - & 1 & 2 & 2 & 3 & 18 & II & 23 & 160 \\
\hline Spirodela polyrrhiza & - & - & - & - & - & 16 & I & 14 & 1 \\
\hline Lemna minor & - & - & 2 & 4 & 3 & 5 & I & 12 & 140 \\
\hline Schoenoplectus lacustris & - & - & - & 1 & - & 12 & I & 11 & 16 \\
\hline Fontinalis antipyretica & - & - & - & 2 & 5 & 3 & I & 9 & 52 \\
\hline Sagittaria sagittifolia & - & - & - & 2 & 1 & 6 & I & 8 & 35 \\
\hline Chara tomentosa & - & - & 1 & 1 & 3 & 2 & I & 6 & 61 \\
\hline Phragmites communis & - & - & - & - & - & 7 & $\mathrm{I}$ & 6 & 1 \\
\hline Alisma plantago-aquatica & - & - & - & 3 & - & 3 & I & 5 & 46 \\
\hline Salvinia natans & - & - & 1 & - & - & 5 & $\mathrm{I}$ & 5 & 33 \\
\hline Chara fragilis & - & - & - & 1 & 1 & 4 & $\mathrm{I}$ & 5 & 20 \\
\hline Bquisetum limosum & - & - & - & - & 1 & 4 & I & 5 & 5 \\
\hline
\end{tabular}

does not occur on habitats with a mineral substrate and in rather rapid water courses where Nuphar luteum forms compact phytocenoses.

Further obtained published and unpublished material greatly augmented the number of phytosociological records available to the author from the group "Myriophyllo-Nupharetum". They are listed in synthetic tables according to the new approach, comprising a total of 1554 phytosociological records, 1361 of which represent the association Nuphareto-Nymphaeetum albae (Table 2), 115 - Myriophylletum verti- 
Table 4

Nymphaeetum candidae Miljan 1958

\begin{tabular}{|c|c|c|c|c|c|c|c|c|}
\hline \multirow{2}{*}{ Species } & \multicolumn{6}{|c|}{ Abundance } & \multirow{2}{*}{ Presence } & \multirow{2}{*}{$\begin{array}{l}\text { Coeffic. } \\
\text { of cover }\end{array}$} \\
\hline & 5 & 4 & 3 & 2 & 1 & + & & \\
\hline \multicolumn{9}{|l|}{ Ch. Nymphaeetum candidae } \\
\hline Nymphaea candida & 16 & 28 & 28 & 6 & - & - & 100 & 5519 \\
\hline \multicolumn{9}{|l|}{ Ch. Nymphaeion } \\
\hline Nuphar luteum & - & - & 4 & 13 & 9 & 12 & III & 543 \\
\hline Potamogeton natans & - & - & 2 & 4 & 12 & 16 & III & 264 \\
\hline Hydrochar1s morsus-ranae & - & - & - & 5 & 6 & 9 & II & 151 \\
\hline Stratiotes aloides & - & - & - & 1 & 2 & 10 & 17 & 36 \\
\hline Nymphaea alba & - & - & - & 1 & 3 & 5 & 12 & 42 \\
\hline Polygonum amphibium $f$. natans & - & - & - & - & 3 & 2 & 6 & 19 \\
\hline Limnanthemum nymphoides & - & - & - & 2 & - & 1 & I & 45 \\
\hline \multicolumn{9}{|l|}{ Ch. Potametea } \\
\hline Ceratophyllum demersum & 2 & 5 & 3 & 1 & 5 & 12 & 36 & 825 \\
\hline Myriophyllum spicatum & - & 1 & - & 3 & 2 & 17 & II & 162 \\
\hline Potamogeton lucens & $=$ & - & 2 & 1 & 5 & 8 & 21 & 151 \\
\hline Blodea canadensis & - & 2 & - & 3 & 1 & 9 & 19 & 235 \\
\hline Batrachium c1roinatum & - & - & - & 1 & 2 & 12 & 19 & 36 \\
\hline Utricularia vulgaris & - & - & - & - & 3 & 7 & 13 & 20 \\
\hline Potamogeton compressus & - & - & - & - & 2 & 6 & 10 & 14 \\
\hline Myriophyllum verticillatum & - & 1 & - & 2 & 1 & 3 & 9 & 131 \\
\hline Potamogeton crispus & - & - & - & - & 2 & 4 & 8 & 13 \\
\hline Potamogeton perfoliatus & - & - & - & - & 1 & 3 & 5 & 7 \\
\hline Potamogeton rutilus & - & - & - & - & - & 3 & 4 & 1 \\
\hline Hottonia palustris & - & - & - & - & - & 3 & 4 & 1 \\
\hline Potamogeton pectinatus & - & - & - & - & 1 & 1 & 3 & 7 \\
\hline Najas marina & - & - & - & - & - & 2 & 3 & 1 \\
\hline Potamogeton obtusifolius & - & - & - & - & - & 2 & 3 & 1 \\
\hline Hippuris vulgaris $f$. sublmersa & - & - & - & - & - & 1 & 1 & 1 \\
\hline Batrachium aquatile & - & - & - & - & - & 1 & 1 & 1 \\
\hline Potamogeton pusillus & - & - & - & - & - & 1 & 1 & 1 \\
\hline Ceratophyllum submersum & - & - & - & - & - & 1 & 1 & 1 \\
\hline Callitriche hamulata & - & - & - & - & - & 1 & 1 & 1 \\
\hline \multicolumn{9}{|l|}{ Accompanying species } \\
\hline Equisetum limosum & - & - & - & - & 8 & 10 & II & 52 \\
\hline Lemna trisulca & - & - & 2 & 3 & - & 9 & 18 & 165 \\
\hline Schoenoplectus lacustris & - & - & - & - & 4 & 10 & 18 & 27 \\
\hline Lemna minor & - & - & - & 1 & 2 & 7 & 13 & 36 \\
\hline Typha latifolia & - & - & - & 1 & 1 & 8 & 13 & 30 \\
\hline Sagittaria sagittifolla & - & 1 & 1 & 2 & 1 & 2 & 9 & 180 \\
\hline Spirodela polyrrhiza & - & - & - & 1 & 1 & 4 & 8 & 29 \\
\hline Fhragmites communis & - & - & - & - & 2 & 4 & 8 & 13 \\
\hline Fontinalis antipyretica & - & - & - & - & 2 & 3 & 6 & 13 \\
\hline Glyceria aquatica & - & - & - & - & 1 & 4 & 6 & 7 \\
\hline Comarum palustre & - & - & - & - & - & 5 & 6 & 1 \\
\hline
\end{tabular}

cillati (Table 3) and 78 Nymphaeetum candidae (Table 4) In the synthetic table with an extended amount of information the figures in the particular abundance intervals denote the incidence of the particular species. In the group of accompanying species only those are mentioned the constancy of which exceeded 5 per cent. As basis in classification of the particular records to the appropriate syntaxon was adopted the floristic dominance of the characteristic species. Phytosociological records in which two or three species belonging to different associations in the new approach (Nuphareto-Nymphaeetum albae, Myriophylletum verticillati, Nymphaeetum candidae) occur in equal quantities were classified to the appropriate syntaxons, after taking into account the dynamics of these 


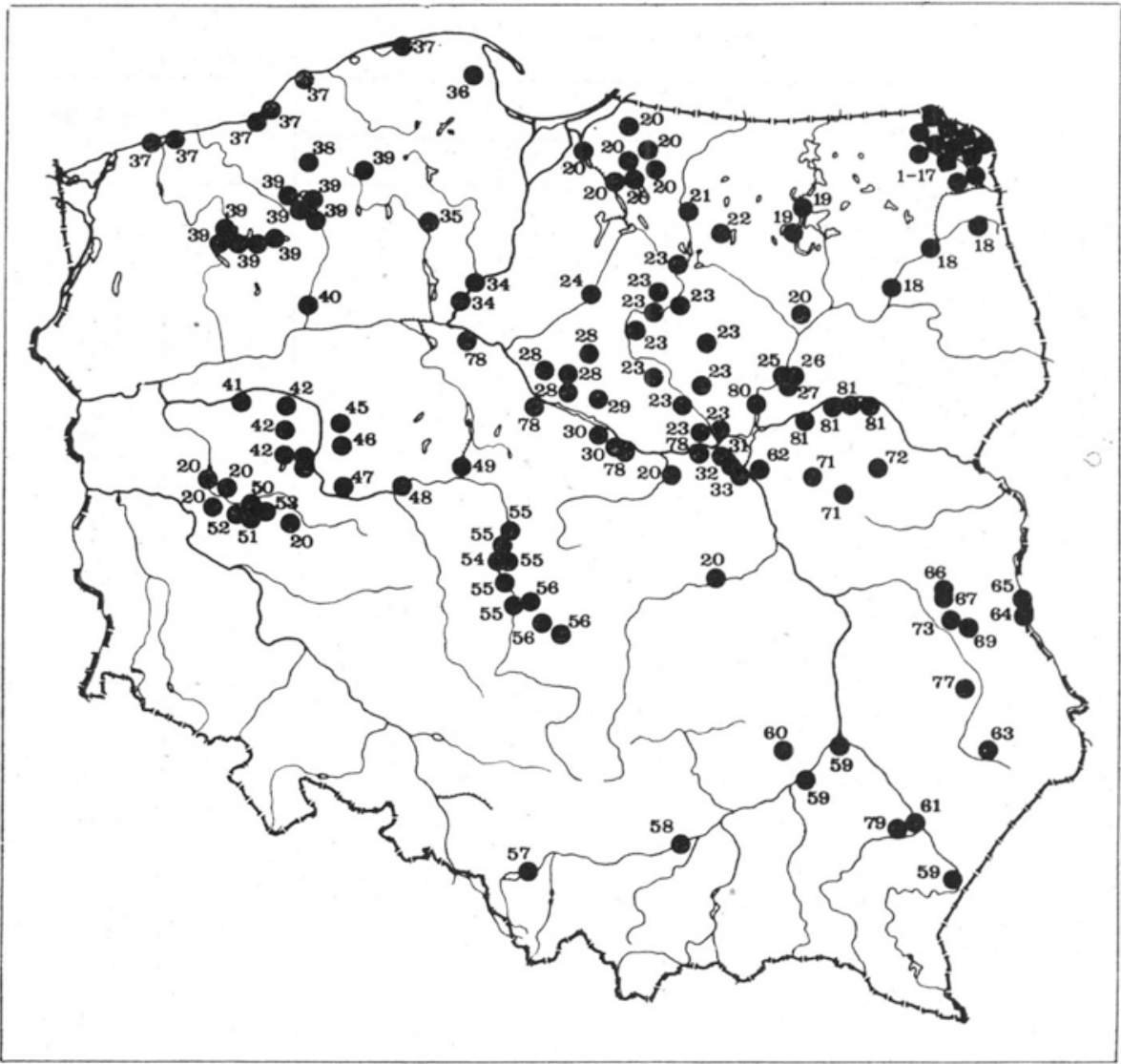

Fig. 1. Sites or groups of sites with Nuphareto-Nymphaeetum albae found in Poland 1 - B e t 1 e wi c z (1974), $2-$ B og d a n ow i c z (1976), 3 - T o m a s z e w i c z (1973-1976 records unpubl.), 4 - Gromadziń ska (1973), $5-\mathrm{K}$ ow a 1 (1973), 6 - Kowalcz y k (1974), $7-$ Rom anowska (1973), $8-$ Poplawska (1975), $9-$ Pospychała $(1975,10-$ K $10-$ Sow s k i (1976), 11 - Kry s z e w s k a-C z yż e w s k a (1974), 12 - J a wor s k a (1974), 13 B or ow a (1975), $14 \neg \mathrm{R}$ udnik (1975), $15-\mathrm{S}$ ob ot ka (1967), $16-\mathrm{S}$ u jecki (1973/74 records unpubl.), 17 - wierzbicka (1973), 18 - Pałczyński (1975), $19-$ Polakowski (1974 records unpubl.), 20 - To mas zewicz (1974-1976 records unpubl.), $21-$ Polkowski, D z i e d zic (1970), 22 - D ziedzic (1973), 23 - Lej m b a ch (1973-1975 records unpubl.), 24 - K ęp czyński, Zielski (1974), 25 - Giniel (1972), 26 - K u bis (1973), 27 - Wiec zorko (1972), 28 - K ęp czyński (1965), 29 - Kęp czyński (1960), $30-$ Tomaszewi c z (1977), $31-$ W i e r z b ow s k a (1971), $32-$ D a wid (1971), $33-$ Gr y c z k a (1969), $34-$ F a bis za k (1974), $35-$ B oi ńs ki, Boińs k a, C e y now a-Gieldon (1974), $36-D$ ąm bS k a (1965), 37 - D ą m b s k a, K r a s k a (1975 records unpubl.), 38 - S p i e w a k o w s ki (1974), $39-$ Michna (1976), $40-\mathrm{Dz}$ iedzic, A sztemborski (1969), $41-\mathrm{D}$ ą mb k a (1961), $42-\mathrm{S}$ taniew s ka-Z ą tek (1972), $43-\mathrm{S}$ ieminiak (1972), 44 - Przybys 1 awska (1972), 45 - Garstkiewicz (1967), 46 - Markiewicz (1972), 47 - Goldy n (1975), $48-$

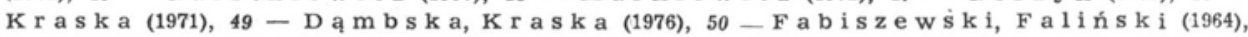
$51-$ Kordus (1974), 52 - J ankow ska (1975), 53 - M i ch a lak (1975), 54 - K r z y wań-

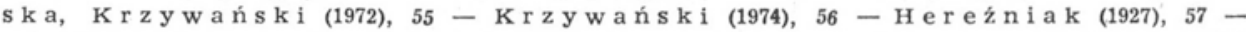
Si eminiak (1976), 58 - D ubiel (1973), 59 - Piór ecki (1975), 60 - O chyra (1976), $61-\mathrm{N}$ owińs ki (1930), $62-\mathrm{Dz}$ ierż a $(1971), 63-\mathrm{Iz}$ de b s k a (1969), $64-\mathrm{F}$ i jalkowSki (1967), 65 - Fijalkowski (1960), 66 - Popiolek (1971), 67 - Popiołek (1974), 68 Popiolek (1973), $69-\mathrm{F}$ ijalkowski (1966), $70-\mathrm{Szczepa}$ á k a (1975), $71-\mathrm{Pod}$ bielkow ski (1968), $72-$ Kord us (1975 records unpubl.), $73-$ Fi ja 1 k ow s ki, Kozak (1970), $74-$ Fijalkowski (1960a), $75-$ Podbielkowski (1969), 76 - Kępczyński, Ceynow a-Gie 1 don (1972), $77-$ B a r y la (1965), $78-$ Kordakow (1974), $79-$ Nowiński (1928), 80 - Tomas zewicz (1969a), 81 - Tom as zewicz (1969), 82 - Podbielkowski 
species and of the community. When the abundance of Nuphar luteum or Nymphaea alba and Myriophyllum verticillatum was equal, the records were included into the table Nuphareto-Nymphaeetum albae. Similarly, when the numbers of Nymphaea candida and Myriophyllum verticillatum were equal, the records were listed in the table Nymphaeetum candidae. In the case of equal quantities of Nuphar luteum and Nymphaea candida the records were classified to the table Nuphareto-Nymphaeetum albae because, as it results from observations and the accumulated material that Nuphar luteum has a wider ecological amplitude and is more dynamic than Nymphaea candida. Records with the above mentioned combinations constitute about 3 per cent of the total number of records. It should be borne in mind that part of these phytosociological records are biassed (as indicated by the water depths given

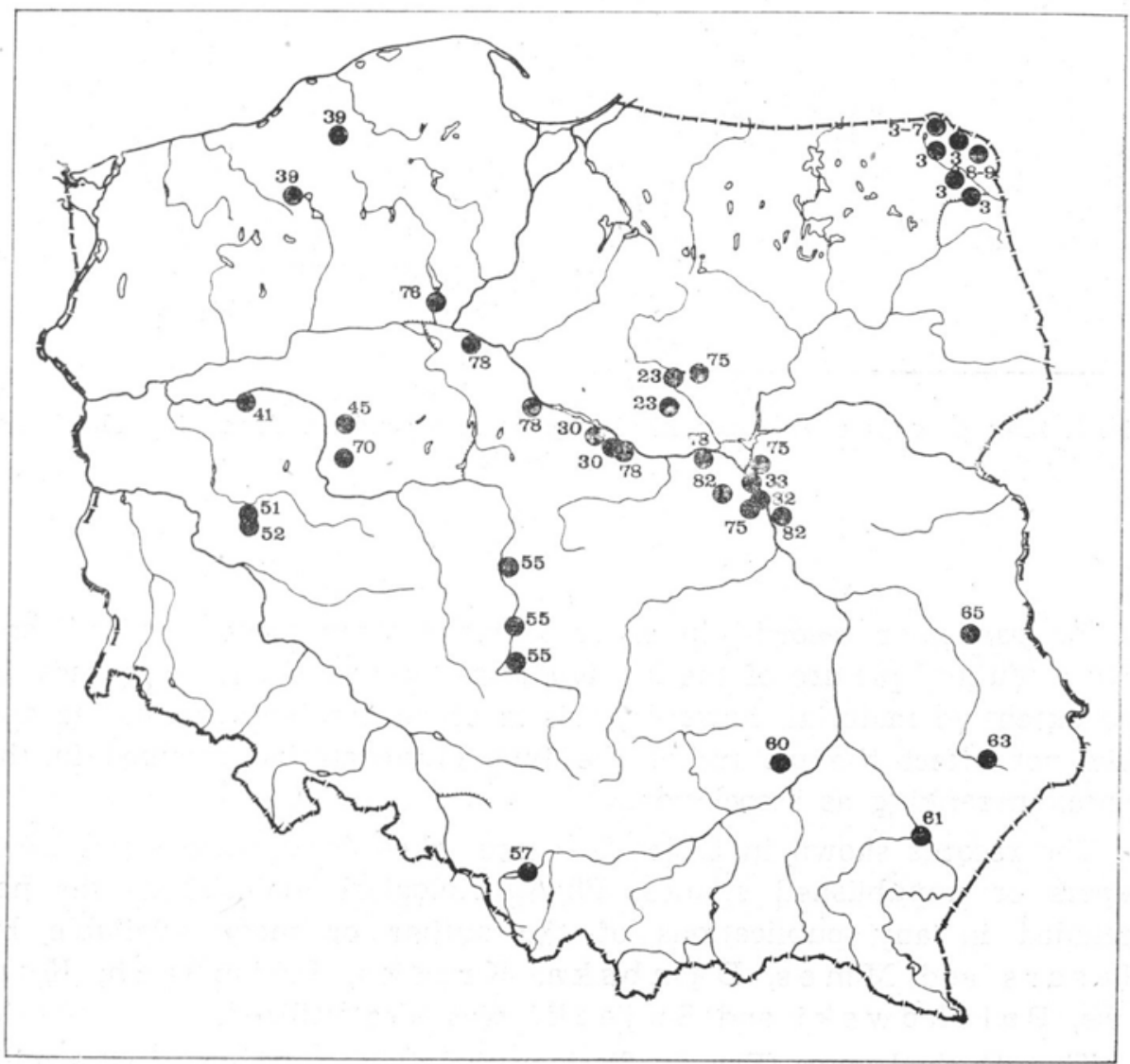

Fig. 2. Sites or groups of sites with Myriophylletum verticillati found in Poland (notations as in fig. 1) 


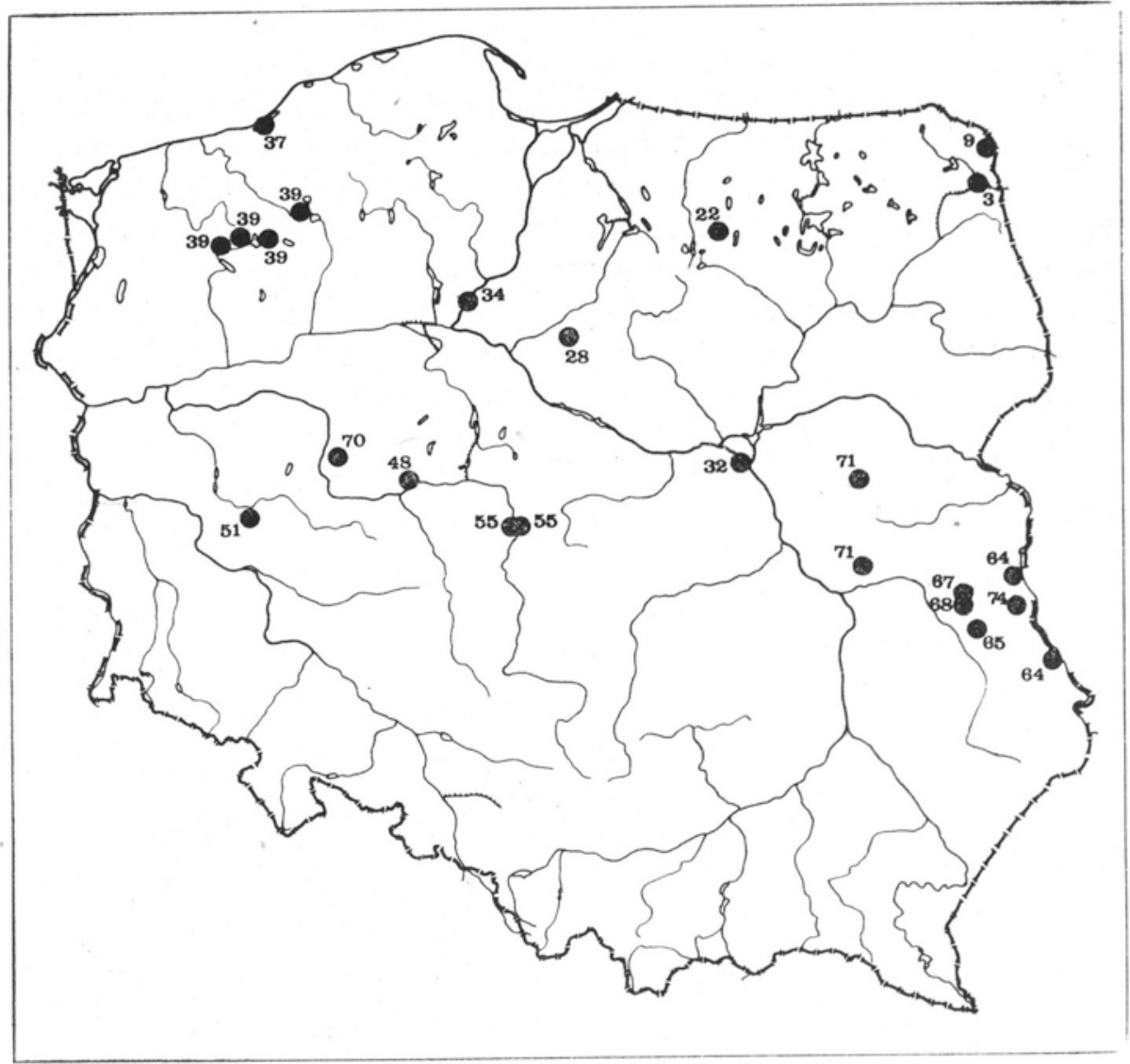

Fig. 3. Sites or groups of sites with Nymphaeetum candidae found in Poland (notations as in fig. 1)

in the particular records) in order to make them more "typical" and give a "fuller" picture of the Myriophyllo-Nupharetum phytocenoses. In the extensive material, however, this is of no greater consequence and does not affect the picture of the type of vegetation grouped in the syntaxon ranking as association.

- The records shown in tables 2-4 are taken from various published papers or unpublished studies. Phytosociological material so far not included in any publications of the author or made available by Messrs and Mmes, Dąmbska, Kraska, Lejmbach, Kor dus, Polakowski and Sujecki was also utilized.

The attached maps (Figs 1-3) show the sites or groups of sites with Nuphareto-Nymphaeetum albae, Myriophylletum verticillati and Nymphaeetum candidae recorded in Poland to date. 


\section{REFERENCES}

B a r y ł a R., 1965. Zbiorowiska roślinne w dolinie rzeki Źółkiewki, Annales UMCS, Sectio E, 19: 229-262.

Betlewicz E., 1974. Roślinność Pojezierza Suwalskiego. Zbiorowiska roślinne i flora Jeziora Przechodniego, Warszawa (M. S.).

B og d a n ow i c z L., 1976. Roślinność i flora jezior: Białego, Czarnego, Płaskiego i Malona na Pojezierzu Sejneńskim, Warszawa (M. S.).

Boiński M., Boińska U., Ce ynowa-Giełdon M., 1974. Roślinność jezior Zdręczno i Kozie na obszarze Borów Tucholskich, Stud. Soc. Scient. Torunensis, sectio $\mathrm{D}, 10,1$ : 1-35.

B or ow a I., 1975. Roślinność Pojezierza Sejneńskiego. Zbiorowiska roślinne i flora jeziora Gieret, Warszawa (M. S.).

D a w id K., 1971. Roślinność wodna starorzeczy lewego brzegu Wisły na odcinku od Burakowa do Cząstkowa Polskiego, Warszawa (M. S.).

D ąmbska I., 1961. Roślinne zbiorowiska jeziorne okolic Sierakowa i Międzychodu, Pozn. Tow. Przyj. Nauk, Wydz. Mat.-Przyr., 23, 4: 1-120.

D ą mbska I., 1965. Roślinność litoralu jezior lobeliowych Pojezierza Kartuskiego. Pozn. Tow. Przyj. Nauk, Wydz. Mat.-Przyr., Prace Kom. Biol. 30, 3: 1-53.

Dąmbska I., Kraska M., 1976. Zbiorowiska roślin naczyniowych w jeziorach konińskich, Wydawnictwa UAM, seria Biol. 6: 25-50, Poznań.

Dubiel E., 1973. Zespoly roślinne starorzeczy Wisły w Puszczy Niepołomickiej i jej otoczeniu, Zakładu Ochrony Przyrody PAN, Studia Naturae, seria A, 7: $67-124$.

Dziedzic J., Asztemborski J., 1969. Roślinność jezior okolic Piły i Smiałowa. Pozn. Tow. Przyj. Nauk., Wydz. Mat.-Przyr., Prace Kom. Biol. 34, 1: 1-56.

Dziedzic J., 1973. Szata roślinna niektórych jezior zachodnich Mazur, Olsztyn (M. S.).

Dzierżak U., 1972. Roślinność starorzeczy Wisły na odcinku Swidry Wielkie Glinki, Warszawa (M. S.).

Fabiszak S., 1974. Zespoły roślinne w dolinie Wisły na odcinku Fordon Swiecie, Toruń (M. S.).

F a biszewski J., Faliński J. B., 1964. O roślinności okolic Przemętu, Przyroda Polski Zach. 8, 1-4: 23-46.

Fijałkowski D., 1960. Szata roślinna jezior Łęczyńsko-Włodawskich i przylegających do nich torfowisk, Annales UMCS, sectio B, 14, 3: 131-206.

Fijałkowski D., 1960a. Stosunki geobotaniczne torfowiska ,Dubeczno" koło Włodawy, Rocznik Nauk Rol., seria A, 80, 3: 449-496.

Fijałk owski D., 1966. Zbiorowiska wodno-torfowiskowe rezerwatu Swierszczów koło Włodawy, Annales UMCS, section C, 20, 12: 179-194.

Fijałkowski D., 1967. Zbiorowiska roślinne lewobrzeżnej doliny Bugu w granicach woj. lubelskiego, Annales UMCS, sectio C, 21, 17: 247-312.

Fijałk owski D., Kozak K., 1970. Roślinność rezerwatu ,Torfowisko nad Jeziorem Czarnym Sosnowickim", Annales UMCS, sectio C, 25: 213-241.

Garstkiewicz K., 1967. Roślinność jezior Skockich: Maciejak, Włókna, Brzeźno na północ od Poznania, Bad. Fizjogr. nad Polską Zach. 20: 59-78.

Giniel 1 B., 1972. Roślinność wodno-bagienna starorzeczy Narwi na odcinku od Czerniaw do rzeki Orzyc. Część zachodnia, Warszawa (M. S.).

G ołdyn R., 1975. Zbiorowiska roślinne Jeziora Raczyńskiego pod Zaniemyślem, Bad. Fizjogr. nad Polską Zach. 28: 49-87.

G r o m a dz ińs k a K., 1973. Roślinność jeziora Kojle, Warszawa (M. S.).

Gryczka T., 1969. Roślinność i flora akwenów lewobrzeżnej Warszawy, Warszawa (M. S.). 
Hereźniak J., 1972. Zbiorowiska roślinne doliny Widawki, Monogr., Bot. 35: $1-160$.

I z d e bska M., 1969. Zbiorowiska roślinne górnego odcinka doliny Wieprza ze szczególnym uwzględnieniem zbiorowisk łąkowych, Fragm. Flor. et Geobot. 15 (3): 283-332.

J a nkowska J., 1975. Roślinność Jeziora Wieleńskiego na Pojezierzu Leszczyńskim, Poznań (M. S.).

J a w or owska W., 1974. Zbiorowiska roślinne i flora Jeziora Okrągłego, Warszawa (M. S.).

K ępczyński K., 1960. Zespoły roślinne Jezior Skępskich i otaczających je łąk, Stud. Soc. Scient. Torunensis, Suppl. 6: 1-244.

Kępczyński K., 1965. Szata roślinna Wysoczyzny Dobrzyńskiej, Toruń.

Kępczyński K., Ceynowa-Giełdon M., 1972. Obserwacje nad roślinnością Zalewu Koronowskiego, Stud. Soc. Scient. Torunensis, sectio D, 9, 4: 1-68.

Kępczyński K., Zielski A., 1974. Zespoły roślinne jeziora Mieliwo i torfowiska do niego przyległego w powiecie brodnickim, Acta Univ. Nicolai Copernici, Biol. 16: 125-167.

Kłosowski St., 1976. Roślinność jeziora Pomorze i Buchta na Pojezierzu Sejneńskim, Warszawa (M. S.).

K och W., 1926. Die Vegetationseinheiten der Linthebene unter Berücksichtigung der Verhältnisse in der Nordostschweiz, Jahrb. d. St. Gall. Naturw. Ges., 61, 2, St. Gallen.

K ordak ow J., 1974. Zarastanie łach i starorzeczy w dolinie Wisły między Modlinem i Toruniem, Poznań (M. S.).

K ordus B., 1974. Zbiorowiska roślinne jezior Przemęckich (Jez. Przemęckiego Północnego, Błotnickiego, Radomierskiego, Olejnickiego), Poznań (M. S.).

K ow a 1 A., 1973. Roślinność jeziora Jaczno, Warszawa (M. S.).

Kowa lczyk J., 1974. Roślinność Pojezierza Północnosuwalskiego. Zbiorowiska roślinne -i flora jeziora Krajwelek, Warszawa (M. S.).

Kraska M., 1971. Zbiorowiska roślin wodnych i błotnych okolic Pyzdr w Pradolinie Warszawsko-Berlińskiej, Bad. Fizjogr. nad Polską Zach., Biol. 24: 181-202.

Kryszewska-Czyżewska J., 1974. Roślinność Pojezierza Północnosuwalskiego. Zbiorowiska roślinne i flora jeziora Gulbin, Warszawa (M. S.).

Krzywańsk'a J., Krzy wánski D., 1972. Zarastanie dołów potorfowych i rowów melioracyjnych w dolinie Warty pod Małkowem i Bartochowem, Zeszyty Nauk. Uniw. Łódz., seria 2, 51: 127-144.

Krzywański D., 1974. Zbiorowiska roślinne starorzeczy środkowej Warty, Monogr. Bot. 43: 1-80.

K u b is E., 1973. Roślinność starorzeczy prawego brzegu Narwi na odcinku Czerniawy - Sielc Stary, Warszawa (M. S.).

Markiewicz H., 1972. Roślinność stawów Kuracz i Zielony Dworek w Promnie pod Poznaniem, Bad. Fizjogr. nad Polską Zach., Biol. 25: 83-114.

M ichalak D., 1975. Roślinność Jeziora Osłonińskiego na Pojezierzu Leszczynskim, Poznań (M. S.).

Michna I., 1976. Roślinne zbiorowiska jeziorne pojezierzy Drawskiego i Bytowskiego, Pozn. Tow. Przyj. Nauk, Prace Kom. Biol. 43: 1-74.

N owiński M., 1928. Zespoły roślinne Puszczy Sandomierskiej. I. Zespoły roślinne torfowisk niskich pomiędzy Chodaczowem a Grodziskiem, Kosmos, seria A, 52, 3-4: 457-546. 
Nowiński M., 1930. Roślinność i znaczenie dla rolnictwa torfowisk niskich z okolic ujścia Wisłoka do Sanu w południowo-wschodniej części dawnej Puszczy Sandomierskiej, PAU, Prace Rolniczo-Leśne, 3: 1-89.

O chyra R., 1976. Zarastanie lejków krasowych w okolicach Staszowa, Kraków (M. S.).

Pałczyński A., 1975. Bagna Jaćwieskie pradoliny Biebrzy, Rocznik Nauk Rol., seria D, 145, PWN, Warszawa.

Piórecki J., 1975. Trapa natans L. w Kotlinie Sandomierskiej (ekologia, rozmieszczenie i ochrona), Rocznik Przemyski 15-16: 347-400.

Podbielkowski Z., 1967. Zarastanie rowów melioracyjnych na torfowiskach okolic Warszawy, Monogr. Bot. 23, 1: 1-171.

Podbielkowski Z., 1968. Roślinność stawów rybnych woj. warszawskiego, Monogr. Bot. 27: 1-123.

Podbielkowski Z., 1969. Roślinność glinianek woj. warszawskiego, Monogr. Bot. 30: 119-156.

Polakowski B., Dziedzic J., 1970. Roślinność naczyniowa Jeziora Kortowskiego, Zeszyty Nauk. WSR w Olsztynie, seria A, Suppl. 3: 1-39.

P opiołek Z., 1971. Roślinność wodna i przybrzeżna jezior okolic Ostrowa Lubelskiego na tle warunków siedliskowych. Część I. Jezioro Kleszczów, Annales UMCS, section C, 26: 387-408.

P opiołek Z., 1973. Roślinność wodna i przybrzeżna jezior okolic Ostrowa Lubelskiego na tle warunków siedliskowych. Część III. Jezioro Miejskie, Annales UMCS, sectio C, 28: 191-213.

P opi ołek Z., 1974. Roślinność wodna i przybrzeżna jezior okolic Ostrowa Lubelskiego na tle warunków siedliskowych. Część IV. Jezioro Czarne Gościnieckie, Annales UMCS 29: 333-353.

Popławska J., 1975. Zbiorowiska roślinne i flora jeziora Wiłkokuk, Warszawa (M. S.).

P ospych ała J., 1975. Roślinność i flora jeziora Kaczan, Warszawa (M. S.).

Prz y b y s ła w s k a H., 1972. Zbiorowiska roślinne Jeziora Rosnowskiego w Wielkopolskim Parku Narodowym, Poznań (M. S.).

R o man owska L., 1973. Roślinność jeziora Perty, Warszawa (M. S.).

Rudnik H., 1975. Roślinność i flora jeziora Dowcień, Warszawa (M .S.).

S e g a l S., 1968. Ein Einteilungsversuch der Wasserpflanzengesellschaften [in]: Pflanzensoziologische Systematik, Berichte über das Internationale Symposium in Stolzenau/Weser 1964, Verlag W. Junk N. V., Den Haag.

Siem in iak D., 1972. Zbiorowiska roślinne Jeziora Góreckiego i jeziora Skrzynka w Wielkopolskim Parku Narodowym, Poznań (M. S.).

Sieminiak D., 1976. Zbiorowiska roślinne stawów rybnych w okolicach Czechowic-Dziedzic, Prace Nauk. Uniw. Śląskiego, 145 (2): 54-67.

S obotka D., 1967. Roślinność strefy zarastania bezodpływowych jezior Suwalszczyzny, Monogr. Bot. 23, 2: 175-258.

Śpiewakowski R., 1974. Badania nad zmianami w szacie roślinnej pod wpływem nierytmicznych wahań poziomów wody w zespole jezior „Elektrowni wodnej Żydowo", Slupsk (M. S.).

Staniewska-Zą tek W., 1972. Zbiorowiska roślinności wodnej w dolinach Samy i Samicy w Wielkopolsce, Bad. Fizjogr. nad Polską Zach. 25: 115-134.

S z c ze pańska M., 1975. Roślinność wodna okolic Nekli, Poznań (M. S).

Tomaszewicz H., 1969. Roślinność wodna i szuwarowa starorzeczy Bugu na obszarze województwa warszawskiego, Acta Soc. Bot. Pol. 38 (2): 217-245.

T o maszewicz H., 1969a. Roślinność wodna Jeziora Zegrzyńskiego, Acta Scc.

Bot. Pol. 38 (3): 401-424. 
T o.m a s ze wicz H., 1977. Roślinność wodno-bagienna w akwenach zlewni Skrwy i ciechomickiej na Pojezierzu Gostynińskim, Monogr. Bot. 52: 1-142.

Wieczorko J., 1972. Roślinność wodno-bagienna starorzeczy Narwi na odcinku od Czerniaw do rzeki Orzyc. Część centralna starorzeczy, Warszawa (M. S.).

W i e rzbicka B., 1973. Roślinność jeziora Kamenduł, Warszawa (M. S.).

W i e r z bowska A., 1971. Roślinność wodna starorzeczy lewego brzegu Wisły na odcinku od Cząstkowa Polskiego do Kazunia Polskiego, Warszawa (M. S.).

Author' address:

Dr Henryk Tomaszewicz

Institute of Botany, Warsaw University; Al. Ujazdowskie 4, 00-478 Warszawa; Poland

Nowe ujęcie syntaksonomiczne fitocenoz Myriophyllo-Nupharetum W. Koch 1926 i ich rozmieszczenie $w$ Polsce

\section{* Streszczenie}

Podawany prawie we wszystkich pracach monograficznych i syntetycznych, dotyczących roślinności wodnej, zespół Myriophyllo-Nupharetum (=Myriophylleto verticillati-Nupharetum), został przez W. K o ch a (1926) opisany bez żadnej dokumentacji materiałowej w postaci zdjęć fitosocjologicznych lub tabel syntetycznych. W.związku z tym w obrębie tej bliżej niesprecyzowanej jednostki umieszczano fitocenozy o zupełnie różnym składzie gatunkowym, różnej dynamice, różnych wymaganiach siedliskowych i amplitudach ekologicznych, a w systemie zbiorowisk roślinnych reprezentujące dwa oddzielne związki (Nymphaeion, Eu-Potamion). Po przeanalizowaniu 1554 zdjęć fitosocjologicznych z terenu Polski, w których gatunkami dominującymi były gatunki uważane za charakterystyczne dla Myriophyllo-Nupharetum (Nuphar luteum, Nymphaea alba, N. candida i Myriophyllum verticillatum) wynikła konieczność nowego ujęcia tych zbiorowisk w trzy oddzielne zespoly: Nuphareto-Nymphaeetum albae, Myriophylletum verticillati i Nymphaeetum candidae. Materiały reprezentujące te syntaksony zostały zestawione w tabele syntetyczne o rozszerzonym zasobie informacji (tab. 2-4), z których jasno wyni$\mathrm{ka}$, iż są to dobrze określone jednostki o randze zespołu. Załączone trzy mapy (ryc. 1-3) przedstawiają rozmieszczenie w Polsce dotychczas stwierdzonych stanowisk lub grup stanowisk wydzielonych zespołów. 